\title{
Hypofractionated radiotherapy recommendations for localized prostate cancer in Brazil
}

\author{
Daniel Moore Freitas Palhares ${ }^{1 *}\left(\mathbb{D}\right.$, Leonardo Cunha Furbino Pimentel ${ }^{2}(\mathbb{D}$, \\ Marcus Simões Castilho ${ }^{2}$ (D), Andréa Barleze da $\operatorname{Costa}^{3}$ (D), Márcio Lemberg Reisner ${ }^{4}$ (D), \\ Felipe Quintino Kuhnen ${ }^{5}$ (D) Anderson Pássaro ${ }^{6}$ (D) Elton Trigo Teixeira Leite $\mathbb{1}^{7}$, \\ Fábio de Lima Costa Faustino 8 (1), Fernando Mariano Obst ${ }^{9}$ (1), \\ Flávio Napoleão Buarque Barbosa Ferro $\operatorname{Costa}^{10} \mathbb{1}^{0}$, Giovani Thomaz Pioner ${ }^{11}$ (D), \\ Ícaro Thiago de Carvalho ${ }^{12}$ (), João Luís Fernandes da Silva ${ }^{13}$ (ㅇ), Lisa Karina Kokay Morikawa ${ }^{14}$ (1), \\ Pedro Henrique da Rocha Zanuncio ${ }^{15}$ (1), Rodrigo de Morais Hanriot ${ }^{16}$ (D), Arthur Accioly Rosa ${ }^{17}$
}

\section{SUMMARY}

OBJECTIVE: Several prospective randomized trials have shown that hypofractionation has the same efficacy and safety as the conventional fractionation in the treatment of localized prostate cancer. There are many benefits of hypofractionation, including a more convenient schedule for the patients and better use of resources, which is especially important in low- and middle-income countries like Brasil. Based on these data, the Brazilian Society of Radiotherapy (Sociedade Brasileira de Radioterapia) organized this consensus to guide and support the use of hypofractionated radiotherapy for localized prostate cancer in Brasil.

METHODS: The relevant literature regarding moderate hypofractionation (mHypo) and ultra-hypofractionation (uHypo) was reviewed and discussed by a group of experts from public and private centers of different parts of Brasil. Several key questions concerning clinical indications, outcomes and technological requirements for hypofractionation were discussed and voted. For each question, consensus was reached if there was an agreement of at least $75 \%$ of the panel members.

\footnotetext{
${ }^{1}$ Hospital Sírio-Libanês - Brasília (DF), Brazil.

${ }^{2}$ Hospital Felício Rocho - Belo Horizonte (MG), Brazil.

${ }^{3}$ Hospital Mãe de Deus - Porto Alegre (RS), Brazil.

${ }^{4}$ Americas Centro de Oncologia Integrado - Rio de Janeiro (RJ), Brazil.

${ }^{5}$ Hospital São José - Criciúma (SC), Brazil.

${ }^{6}$ Hospital da Providência - Apucarana (PR), Brazil

`Universidade de São Paulo, Hospital Vila Nova Star, Rede D’Or, Instituto do Câncer do Estado de São Paulo - São Paulo (SP), Brazil.

${ }^{8}$ Hospital de Amor de Barretos - Barretos (SP), Brazil.

${ }^{9}$ Grupo Oncoclínicas, Hospital São Lucas Pontifícia Universidade Católica do Rio Grande do Sul - Porto Alegre (RS), Brazil.

${ }^{10}$ Hospital São Domingos - São Luís (MA), Brazil.

${ }^{11 S a n t a}$ Casa de Misericórdia, Hospital Moinhos de Vento - Porto Alegre (RS), Brazil.

${ }^{12}$ Hospital Israelita Albert Einstein - São Paulo (SP), Brazil.

${ }^{13}$ Hospital Sírio-Libanês - São Paulo (SP), Brazil.

${ }^{14}$ Grupo Oncologia D’Or, Clínica São Vicente - Rio de Janeiro (RJ), Brazil.

${ }^{15}$ Hospital Beneficência Portuguesa - São Paulo (SP), Brazil.

${ }^{16}$ Hospital Alemão Oswaldo Cruz - São Paulo (SP), Brazil.

${ }^{17}$ Sociedade Brasileira de Radioterapia, Grupo Oncoclínicas - São Paulo (SP), Brazil.

*Corresponding author: sbradioterapia@sbradioterapia.com.br

Conflicts of interest: the authors declare there are no conflicts of interest. Funding: none.

Received on July 09, 2020. Accepted on January 06, 2021.
} 
RESULTS: The recommendations are described in this article.

CONCLUSION: This initiative will assist Brazilian radiation oncologists and medical physicists to safely treat localized prostate cancer

patients with hypofractionation.

KEYWORDS: Prostatic neoplasms. Radiotherapy. Radiation dose hypofractionation. Consensus.

\section{INTRODUCTION}

Prostate cancer (PCa) is one of the most incident cancers in Brasil and worldwide, representing more than $30 \%$ of all cases in men $^{1,2}$. Most patients are diagnosed with prostate-limited disease and are candidates to curative treatment. Radiotherapy (RT) and surgery are equally effective treatment options. About one third of patients receive definitive $\mathrm{RT}^{3-5}$. Traditionally, patients have been treated with 35 to 44 daily consecutive fractions of 1.8-2.0 Gy (total doses of 70-79.2 Gy) ${ }^{3,5}$. This schedule is known as the conventional fractionated RT (convFx) $)^{3,6-8}$.

In the past few years, many prospective randomized controlled trials (RCT) have shown that the use of moderate hypofractionated (mHypo, fraction sizes between 2.4 and 3.4 Gy) or ultra-hypofractionated RT (uHypo, fraction sizes $>5 \mathrm{~Gy}$ ) has comparable efficacy and toxicity to the convFx ${ }^{9-24}$.

Several reasons might justify these studies. First, some data suggested that hypofractionation could increase the therapeutic ratio, given that $\mathrm{PCa}$ cells are especially sensitive to higher daily radiation doses (low alpha-beta ratio) ${ }^{25}$. This radiobiological advantage would be further translated into clinical advantages. Secondly, higher daily doses mean fewer treatment days. This may benefit patients, the radiation oncology (RO) department, and the public health system: the smaller number of patient's visits to the clinic may reduce logistical challenges, increase patient's adherence and reduce the treatment costs; from the RO department and health system perspective, mHypo and uHypo may increase machine capacity due to the shorter treatment schedule and increased turnover. This is especially important in low and middle income countries (LMIC), where the majority of the population depends on the public health system and where there is a shortage of linear accelerators slots ${ }^{6,26-29}$.

Although hypofractionation is an important strategy for PCa treatment, its implementation in LMIC might be a challenge because of the need for more intensive staff training and for higher technology upgrades, especially in non-academic community centers.

In order to support radiation oncologists and physicists to implement hypofractionation in the clinical practice, this consensus aimed to guide indications and the minimum requirements to safely conduct hypofractionation RT for localized PCa patients in Brasil. It does not address hypofractionation in patients with clinically positive lymph nodes or those that underwent prior prostatectomy.

\section{METHODS}

The Brazilian Society of Radiotherapy (Sociedade Brasileira de Radioterapia - SBRT) designated a group of seven radiation oncologists to prepare and conduct a consensus meeting that took place in the city of São Paulo/SP on October 11, 2019. Sixteen radiation oncologists from different areas of Brasil, from both public and private institutions, with known expertise in the topic, attended the meeting and composed the panel. One urologist and one medical physicist were invited to represent the Brazilian Urology Society (Sociedade Brasileira de Urologia - SBU) and the Brazilian Association of Medical Physicists (Associação Brasileira de Física Médica - ABFM), respectively. They could make suggestions and present their opinion, but they could not vote.

A systematic literature review was carried out in MEDLINE PubMed using the PICO (Population, Intervention, Comparison, and Outcome) model. We reviewed studies including men with localized PCa that were treated with hypofractionated or ultra-hypofractionated RT. The outcomes of interest were PCa control, overall survival, acute and late toxicity, and quality of life. RCTs, meta-analyses of RCTs, or selected prospective observational studies published in English between December 1, 2001 and August 31, 2019 were evaluated. Papers addressing postoperative radiation (adjuvant or salvage treatment), brachytherapy, metastatic disease, or re-irradiation were excluded.

The voting methodology followed this fashion. First, a formal procedure was established before voting. The reviewed papers were discussed to support radiation oncologists to take their most informed decision before each key question (KQ). Time was given for members to show if they were in favor or against each statement. After arguments, the voting was conducted according to the Delphi Method ${ }^{30}$. The panelists could 
agree or disagree in each KQ. The consensus was reached if there was an agreement of at least $75 \%$ of the panel members. Additional commentaries or particular recommendations could be collected after voting based on panel discussion and agreement.

The grade for recommendation was suggested based on the scientific evidence level, which was qualified as strong, medium, or weak, as follows:

- Strong level - multiple concordant randomized controlled trials (RCTs) and/or of a robust meta-analysis of RCTs.

- Medium level - less robust meta-analysis, a single RCT or observational non-randomized trials.

- Weak level - consensual opinions of experts.

The meeting was divided into two sections that included topics about mHypo and uHypo.

\section{Key questions and recommendations}

\section{Section I - Moderate hypofractionation in localized prostate cancer}

\section{1 - Optimal scenario for the indication}

KQ 1 - Table 1. In which of the following cancer risk groups is the use of mHypo adequate?

\section{I.1.1 - Impact of risk stratification group}

The panel considered mHypo to be safe and effective in patients with PCa, regardless the risk group (level of agreement - 100\% for each PCa risk group, level of evidence - strong).
Although there is less evidence to support mHypo for patients with very high-risk features (14), the panel considered it appropriate as long as the dose constraints of organs at risk (OAR) are strictly followed. The indication of mHypo might be independent of the hormone therapy use.

The largest randomized controlled trials that support the scientific evidence for this consensus are the $\mathrm{CHHiP}^{9,10}$, HYPRO $^{11-13}$, PROFIT ${ }^{14}$, RTOG $0415^{15,16}$, FOX CHASE ${ }^{17,18}$, MD Anderson ${ }^{19-21}$, and Italian trials ${ }^{22}$. Taken together, more than 6,000 patients were enrolled. They demonstrate that at a 5-6-year follow-up the mHypo presents oncological outcomes similar to convFx. More than $80 \%$ of the enrolled patients were at low or intermediate risk. Thus, the scientific evidence is very strong for these patient groups. High-risk patients were also well represented (about 1,000 patients), and there is no reason to believe that tumor control will be inferior in high or very high-risk patients treated with mHypo.

KQ 2 - Table 1. Is the use of mHypo adequate in the following situations?

\subsection{2 - Impact of seminal vesicles}

Although it is not the objective of this consensus to select which patients should or should not have the seminal vesicles treated, all agreed that it is appropriate to use mHypo when including the seminal vesicles (level of agreement $-100 \%$, level of evidence - strong).

\subsection{3 - Impact of pelvic lymph node drainage}

The group agreed that the use of mHypo is appropriate when the physician decides to electively treat the pelvic drainage (level of agreement $-88 \%$, level of evidence - medium). However, the

Table 1. Optimal scenario for the indication of moderate hypofractionation.

\begin{tabular}{|c|c|c|c|}
\hline Scenarios & $\begin{array}{c}\text { Agree, adequate } \\
(\%)\end{array}$ & $\begin{array}{l}\text { Disagree, } \\
\text { inadequate }(\%)\end{array}$ & $\begin{array}{l}\text { Consensus } \\
\text { achieved }\end{array}$ \\
\hline Low risk & 100 & 0 & Yes \\
\hline Intermediate favorable risk & 100 & 0 & Yes \\
\hline Intermediate unfavorable risk & 100 & 0 & Yes \\
\hline High and very high risk & 100 & 0 & Yes \\
\hline $\begin{array}{l}\text { When the radiation oncologist decides to include the } \\
\text { seminal vesicles in the treatment volume }\end{array}$ & 100 & 0 & Yes \\
\hline $\begin{array}{l}\text { When the radiation oncologist decides to include the } \\
\text { pelvic lymph nodes in the treatment volume }\end{array}$ & 88 & 12 & Yes \\
\hline Patients with history of transurethral resection of prostate & 81 & 19 & Yes \\
\hline $\begin{array}{l}\text { Patients with important urinary obstruction disease } \\
\text { (*High IPSS) }\end{array}$ & 12 & 88 & Yes \\
\hline
\end{tabular}

IPSS: international prostate symptom score. *High IPSS: above 18 points. 
panel strongly recommended the use of intensity modulated RT (IMRT) or volumetric modulated arc therapy (VMAT) with image-guided RT (IGRT) in this context.

The Fox Chase trial ${ }^{17,18}$ enrolled 303 high-risk patients and treated the pelvic lymph nodes. The study showed a similar toxicity between mHypo and convFx.

\subsection{4 - Impact of transurethral resection of the prostate}

The panel considered that mHypo is adequate in patients who underwent the transurethral resection of the prostate TURP (level of agreement $-81 \%$, level of evidence - strong). However, they recommended to wait between six and eight weeks for patient's recovery before starting $\mathrm{RT}^{31,32}$.

\subsection{5 - Impact of urinary function}

The panel agreed that patients with severely impaired urinary function should not be treated with mHypo, as they may be subject to increased urinary toxicity (level of agreement $88 \%$, level of evidence - strong). In such cases, they might be referred to symptomatic treatment, either with TURP or pharmacological treatment, before receiving RT. Selecting the patient that should receive symptomatic treatment before RT may be a matter of debate, however, the panel suggests that an International Prostate Symptom Score (IPSS) $\geq 18$ could be used to guide this decision. After symptom relief, mHypo may be applied.

\section{I.2 - Tumor control, toxicity, and quality of life}

KQ 3 - Table 2. How does the mHypo is compared to the conventional fractionation in terms of tumor control, toxicity, and quality of life?

\subsection{1 - Tumor control}

The panel agreed that mHyo is equivalent to convFx in terms of tumor control (level of agreement $-100 \%$, level of evidence - strong). The largest randomized controlled trials that compared mHypo to convFx showed equivalent biochemical control for both arms, with a median follow-up of five to six years ${ }^{9}, 13,14,17,18,21,22$.

\subsection{2 - Toxicity}

The panel considered the use of mHypo to be safe regarding the risks of gastrointestinal (GI) and genitourinary (GU) acute or chronic toxicity (level of agreement $-100 \%$, level of evidence - strong).

Some observations were pointed out. First, randomized controlled trials showed no difference in the risk of acute/late GU or late GI toxicity with mHypo or convFx. However, mHypo was associated with a slightly increase in moderate acute GI toxicity in the CHHiP, PROFIT, HYPRO and Fox Chase tri$\mathrm{als}^{9,11-22}$. Some of the mHypo studies tested dose escalation on the experimental arms, which could justify the increase in acute GI toxicity. The panel, then, considered that the clinical impact of mHypo on this toxicity is small enough that does not compromise this strategy. Secondly, patients with high IPSS score require counseling regarding the risk of increased urinary toxicity, as described before.

The panel agreed that mHypo is safe regarding erectile dysfunction (level of agreement - 100\%, level of evidence - strong).

\subsection{3 - Quality of life}

The panel agreed that mHypo is equivalent to the convFx in terms of quality of life (level of agreement $-100 \%$, level

Table 2. Disease control, toxicity, and quality of life of moderate hypofractionation.

\begin{tabular}{l|c|c|c}
\hline $\begin{array}{l}\text { Outcomes } \\
\text { In terms of disease control, the results of mHypo are similar } \\
\text { to conventional fractionation }\end{array}$ & Agree (\%) & Disagree (\%) & Consensus \\
\hline $\begin{array}{l}\text { In terms of acute urinary toxicity, it is safe to use } \\
\text { moderate hypofractionation }\end{array}$ & 100 & 0 & Yes \\
\hline $\begin{array}{l}\text { In terms of late urinary toxicity, it is safe to use } \\
\text { moderate hypofractionation }\end{array}$ & 100 & 0 & Yes \\
\hline $\begin{array}{l}\text { In terms of acute gastrointestinal toxicity, it is safe to use } \\
\text { moderate hypofractionation }\end{array}$ & 100 & 0 & Yes \\
\hline $\begin{array}{l}\text { In terms of late gastrointestinal toxicity, it is safe to use } \\
\text { moderate hypofractionation }\end{array}$ & 100 & 0 & Yes \\
\hline $\begin{array}{l}\text { In terms of erectile disfunction, it is safe to use } \\
\text { moderate hypofractionation }\end{array}$ & 100 & 0 & Yes \\
\hline $\begin{array}{l}\text { In terms of quality of life, moderate hypofractionation is similar } \\
\text { to conventional fractionation }\end{array}$ & 100 & 0 & Yes \\
\hline
\end{tabular}


of evidence - strong). Data from different studies, including CHHip and RTOG 0415 trials, showed that quality of life in bowel, urinary, and sexual domains was equivalent in patients treated with mHypo or convFx $x^{15,16,18,19,33}$.

\section{I.3 - Preferred schedule}

KQ 4. Is there any preferred scheme for mHypo?

In cases of prostate \pm seminal vesicles treatment, the panelists agreed that the preferred scheme for mHypo is $60 \mathrm{~Gy}$ in 20 fractions of 3 Gy based on the CHHip and PROFIT trials (level of agreement $-100 \%$, level of evidence - medium) ${ }^{9,10,14}$. Together, these two trials included the highest number of patients treated with a single fractionation (more than 2,800 patients included, 1,600 of whom were treated with $20 \times 3 \mathrm{~Gy}$ ) and included representatives from all the risk groups (low, intermediate, and high). Although the panelist recommend this particular schedule, other fractionations evaluated in RCT may also be used ${ }^{13,15,17,18,21,22}$, with the exception of $19 \times 3 \mathrm{~Gy}$, considering it has not been proved to be non-inferior to convFx in the CHHiP trial ${ }^{9,10}$.

In cases of pelvic drainage treatment, the panelist did not agree about any particular fractionation (level of agreement $-50 \%$, level of evidence - medium). Thus, for this situation, each physician should decide the best schedule based on the available RCTs. When opting to treat the pelvic drainage, the panel considered essential to use IMRT/VMAT and IGRT.

\section{4 - Treatment techniques}

KQ 5 - Table 3. Is it adequate to perform mHypo with the following techniques?

\subsection{1 - Conventional or bidimensional radiotherapy}

The panel agreed that the use of conventional or bidimensional RT (2D-RT) is inappropriate to deliver mHypo (level of agreement $-100 \%$, level of evidence - strong). First, none of the prospective studies of mHypo used bidimensional RT. Secondly, using 2D-RT could expose patients to unacceptable toxicity.

\subsection{2 - Conformal or three-dimensional radiotherapy}

The panel considered that the use of conformal or three-dimensional RT (3D-RT) is appropriate to deliver mHypo (level of agreement $-88 \%$, level of evidence - high).

They argued that some RCTs allowed 3D-RT with acceptable toxicity for both convFx and mHypo. For example, all

Table 3. Techniques of moderate hypofractionation treatment.

\begin{tabular}{|c|c|c|c|}
\hline Treatment techniques & $\begin{array}{l}\text { Yes, adequate } \\
(\%)\end{array}$ & $\begin{array}{c}\text { No, } \\
\text { inadequate } \\
(\%)\end{array}$ & $\begin{array}{l}\text { Consensus } \\
\text { achieved }\end{array}$ \\
\hline Planning: conventional or bidimensional radiotherapy & 0 & 100 & Yes \\
\hline Planning: conformal or tridimensional radiotherapy & 88 & 12 & Yes \\
\hline Planning: intensity modulated techniques (IMRT or VMAT) & 100 & 0 & Yes \\
\hline $\begin{array}{l}\text { Modality of IGRT: tridimensional imaging (cone beam computed } \\
\text { tomography; ultrasonography) with or without fiducials }\end{array}$ & 100 & 0 & Yes \\
\hline $\begin{array}{l}\text { Modality of IGRT: online bidimensional imaging (portal) with } \\
\text { fiducials (onboard imaging, EPID) }\end{array}$ & 100 & 0 & Yes \\
\hline $\begin{array}{l}\text { Modality of IGRT: online bidimensional imaging (portal) without } \\
\text { fiducials (onboard imaging, EPID) }\end{array}$ & 69 & 31 & Yes \\
\hline $\begin{array}{l}\text { Modality of IGRT: offline bidimensional imaging (portal) with } \\
\text { fiducials (analogic portal film or electronic portal film without } \\
\text { onboard device) }\end{array}$ & 25 & 75 & Yes \\
\hline $\begin{array}{l}\text { Modality of IGRT: offline bidimensional imaging (portal) without } \\
\text { fiducials (analogic portal film or electronic portal film without } \\
\text { onboard device) }\end{array}$ & 19 & 81 & Yes \\
\hline Frequency of IGRT: once a week & 18 & 82 & Yes \\
\hline Frequency of IGRT: three times per week & 75 & 25 & Yes \\
\hline $\begin{array}{l}\text { Frequency of IGRT: daily for the first five fractions, and then } \\
\text { once a week }\end{array}$ & 82 & 18 & Yes \\
\hline Frequency of IGRT: daily & 100 & 0 & Yes \\
\hline
\end{tabular}

IMRT: intensity modulated radiotherapy; VMAT: volumetric modulated arc therapy; ICRT: image-guided radiotherapy; EPID: electronic portal imaging device. 
patients in the Italian trial ${ }^{22}, 60 \%$ in the CHHip trial ${ }^{9}, 21 \%$ in the RTOG $0415^{15}$ and $5 \%$ in the Hypro ${ }^{13}$ were treated with 3D-RT. The PROFIT trial ${ }^{14}$ also allowed 3D-RT.

The 3D-RT in the CHHiP trial was performed with a complex arrangement of fields (field-in-field technique) in order to improve dose distribution and follow dose constraints?

The panel pointed out, however, that 3D-RT might be used if constraint requirements are met for OAR and planning target volume (PTV) coverage. Although 3D-RT is an acceptable option, the use of IMRT/VMAT, as later discussed, should be encouraged.

\subsection{3 - Intensity modulated techniques}

The panel recommended the use of IMRT/VMAT to deliver mHypo since it is the most appropriate technique to spare OARs, while properly covering the PTV (level of agreement $100 \%$, level of evidence - strong). One might note that the majority of phase III RCTs of mHypo used IMRT only, or strongly encouraged it over 3D-RT ${ }^{9,11-21}$. Similarly, we agreed that IMRT/VMAT is the best option for PCa and should be used, if available.

There is evidence showing lower toxicity with IMRT compared to 3D-RT. For instance, one prospective randomized trial directly compared both techniques for patients treated with 70 Gy/25 fractions ${ }^{34}$. Such study identified lower GU/GI toxicity in the IMRT arm, with similar biochemical control in five years. Additionally, other non-randomized studies employing convFx schedules showed reduced GI/GU toxicity of IMRT over 3D-RT ${ }^{35-37}$.

\section{I.5 - Image-guided radiotherapy}

KQ 6 - Table 3. Considering the use of IGRT, which of the following techniques and frequencies of imaging are adequate for mHypo?

Regarding the technique, the panel considered that both the use of tridimensional imaging devices (cone beam computed tomography-CB-CT; or ultrasonography - US) with or without fiducials or bidimensional online devices with fiducials are adequate to treat patients with mHypo (level of agreement $100 \%$, level of evidence - high). Although the majority of RO considered online bidimensional imaging without fiducials adequate, there was no consensus (level of agreement - 69\%, level of evidence - medium). Finally, offline bidimensional imaging with (level of agreement - 75\%, level of evidence - medium) or without fiducials (level of agreement $-81 \%$, level of evidence - medium) was considered inadequate due to uncertainties concerning quality control of the revealed images and the risk of patient movement during the elapsed time while waiting for film development.
The panel considered inappropriate to image the patient only once a week because data will be insufficient to evaluate systematic setup errors (level of agreement $-82 \%$, level of evidence - medium). However, they agreed that imaging the patient at least three times a week (level of agreement $-75 \%$, level of evidence - medium) or daily for the first five fractions and, therefore, once a week (level of agreement $-82 \%$, level of evidence-medium), or daily throughout the entire treatment is appropriate (level of agreement $-100 \%$, level of evidence - medium).

Once different modalities of IGRT are available, we suggest that each department evaluates the association between technology and number of patients under treatment to adjust IGRT in terms of modality and frequency, considering the recommendations previously given.

The panel also recommended that each department should evaluate set-up and intrafraction errors to better select safe PTV margins, especially for those who perform less-than-daily imaging. They argued that daily imaging should be considered when using very restricted PTV margins. In addition, they recommended that an initial set of images should be evaluated before moving to a less-than-daily frequency.

IGRT may reduce the risk of missing the target and of toxicity, especially for hypofractionated treatments. Its benefit has been demonstrated in studies that compared IGRT to non-IGRT treatments ${ }^{38-46}$. A recently published phase III trial showed that daily image (78\% CB-CT, 22\% 2D-images with fiducials) significantly improved biochemical control and rectal toxicity compared to weekly IGRT for $\mathrm{PCa}^{38}$.

Different forms and frequencies of IGRT were used on phase III RCT, including daily images, such as US, CB-CT or KV associated with fiducial markers ${ }^{13,14}$ or electronic portal imaging device (EPID) for three consecutive images followed by weekly images ${ }^{9,22}$.

\section{Section II - Ultra-hypofractionation}

\section{1 - Optimal scenario for the indication}

KQ 7 - Table 4. In which of the following cancer risk groups is the use of uHypo adequate?

\section{II.1.1 - Risk group stratification impact}

The panel considered uHypo to be safe and effective in patients with low (level of agreement - 100\%, level of evidence - strong), intermediate favorable (level of agreement $-100 \%$, level of evidence - strong), and intermediate unfavorable risk group (level of agreement $-94 \%$, level of evidence - medium). The panel agreed that uHypo is not appropriate for high and very high-risk patients (level of agreement $-81 \%$, level of evidence - medium). 
There are two published phase III RCT studies comparing uHypo to convFx or mHypo. The PACE-B trial ${ }^{24}$ included $7 \%$ low risk and $93 \%$ intermediate favorable risk patients, while the HYPO-PC-RT trial ${ }^{23}$ included $89 \%$ intermediate risk and only $11 \%$ high-risk patients. Other several prospective non-randomized studies of uHypo were published, the majority of them included low to intermediate risk patients ${ }^{47-51}$. Therefore, considering the few number of high-risk patients treated with uHypo, the panel deemed the evidence to not be robust enough to suggest it in the clinical practice. Some panelists that were in favor of using uHypo for high-risk patients pointed out that uHypo should be considered appropriate when one chooses not to irradiate the pelvic lymph nodes. However, this particularity was not submitted for voting and we cannot offer an agreement level. The panel also agreed that the indication of uHypo might be independent of the use of hormone therapy.

KQ 8 - Table 4. Is the use of uHypo adequate in the following situations?

\subsection{2 - Extracapsular disease impact}

The majority of participants considered that the use of uHypo is not appropriate for patients with extracapsular spread (cT3a), although there was no consensus on it (level of agreement $62 \%$, level of evidence - medium). Only few patients with extracapsular spread were treated in the published papers ( $4 \%$ in the HYPO-PC-RT trial ${ }^{23}$ and none in the PACE-B trial ${ }^{24}$ ), which is not enough to support uHypo in this context.

\subsection{3 - Seminal vesicles impact}

Although it is not the objective of this consensus to select the patients that should or should not have the seminal vesicles treated, all agreed that it is appropriate to use uHypo when the physician decides to electively treat the seminal vesicles (level of agreement $-100 \%$, level of evidence - medium).

\section{II.1.4 - Pelvic lymph node drainage impact}

The group agreed that the use of uHypo is not appropriate to treat the pelvic drainage (level of agreement $-100 \%$, level of evidence - low), considering that none of the published RCTs have done it. However, there are ongoing trials evaluating this issue (NCT01953055 and NCT03253978).

\section{II.1.5 - Impact of transurethral resection of the prostate}

There was no consensus if the use of uHypo is adequate or not in patients that underwent TURP (level of agreement - 50\%, level of evidence-low). Therefore, we recommend that each case should be carefully evaluated, and other fractionation options (convFx or mHypo) should be considered for these patients.

\section{II.1.6 - Urinary function impact}

The panel agreed that patients with severely impaired urinary function should not be treated with uHypo as they may be subject to increased urinary toxicity (level of agreement $-100 \%$, level of evidence - low). In such cases, they might be referred to symptomatic treatment before receiving RT with other fractionation schemes (convFx or mHypo).

Table 4. Optimal scenario for the indication of ultra-hypofractionation.

\begin{tabular}{|c|c|c|c|}
\hline Scenarios & Agree, adequate (\%) & $\begin{array}{l}\text { Disagree, } \\
\text { inadequate }(\%)\end{array}$ & Consensus achieved \\
\hline Low risk & 100 & 0 & Yes \\
\hline Intermediate favorable risk & 100 & 0 & Yes \\
\hline Intermediate unfavorable risk & 94 & 6 & Yes \\
\hline High and very high risk & 19 & 81 & Yes \\
\hline Extracapsular disease & 38 & 62 & No \\
\hline $\begin{array}{l}\text { When the radiation oncologist decides } \\
\text { to include the seminal vesicles in the } \\
\text { treatment volume }\end{array}$ & 100 & 0 & Yes \\
\hline $\begin{array}{l}\text { When the radiation oncologist decides } \\
\text { to include the pelvic lymph nodes in the } \\
\text { treatment volume }\end{array}$ & 0 & 100 & Yes \\
\hline $\begin{array}{l}\text { Patients with history of transurethral } \\
\text { resection of prostate }\end{array}$ & 50 & 50 & No \\
\hline $\begin{array}{l}\text { Patients with important urinary obstruction } \\
\text { disease (*High IPSS) }\end{array}$ & 0 & 100 & Yes \\
\hline
\end{tabular}

IPSS: international prostate symptom score. *High IPSS: above 18 points. 


\section{II.2 - Tumor control, toxicity, and quality of life} KQ 9 - Table 5. How does uHypo is compared to the convFx in terms of tumor control, toxicity, and quality of life?

\section{2.1 - Tumor control}

The panel agreed that uHypo is equivalent to convFx in terms of tumor control (level of agreement $-100 \%$, level of evidence strong). The most important phase III RCT that supports this statement is the HYPO-RT-PC trial ${ }^{23}$, which demonstrated after a five-year follow-up that uHypo (7 x $6.1 \mathrm{~Gy})$ was noninferior to convFx (39 x 2 Gy) regarding failure-free survival.

\section{II.2.2 - Toxicity}

The panel considers the use of uHypo to be safe in terms of risk of GI and GU acute or chronic toxicity (level of agreement $-100 \%$ for each statement, level of evidence - strong).

The HYPO-PC-RT trial ${ }^{23}$ showed that early side-effects were slightly higher in the uHypo group. The authors suggested this difference might be a reflection of the accelerated course of uHypo schedule (dose delivered in 2.5 versus 8 weeks in the convFx arm). The physician- and patient-reported late GU/GI toxicities were similar in both fractionation groups, with the exception of increased urinary frequency in the uHypo group one year after treatment. On the other hand, the PACE-B trial $^{24}$ showed no difference in acute GU/GI grade 2 toxicity between convFx/mHypo or uHypo groups. Late toxicity data have not been published for this trial yet.

The panel agreed that uHypo is safe regarding erectile dysfunction (level of agreement $-100 \%$, level of evidence - strong).
The HYPO-PC-RT ${ }^{23}$ reported no significant differences in erectile function between both treatment arms.

\subsection{3 - Quality of life}

The panel agreed that uHypo has evidence supporting a favorable toxicity profile with minimal impact on long-term urinary and bowel quality of life (level of agreement $-100 \%$, level of evidence - strong).

\section{II.3 - Preferred dose schedules}

KQ 10. Is there any preferred scheme for uHypo?

The consensus agreed that these fractionation schemes are preferred for uHypo: 5 x 7.25 Gy (level of agreement - 94\%, level of evidence - medium), $5 \times 8$ Gy (level of agreement $-75 \%$, level of evidence - medium) and 7 x $6.1 \mathrm{~Gy}$ (level of agreement $100 \%$, level of evidence - medium). The experimental arms of the PACE- ${ }^{24}$ and HYPO-PC-RT ${ }^{23}$ trials were, respectively, 36.25 Gy in five fractions of 7.25 Gy and 42.70 Gy in seven fractions of 6.1 Gy. Data from these studies on safety and efficacy were previously discussed. The acceptance of $40 \mathrm{~Gy}$ in five fractions of 8 Gy was based on a single-arm phase II trial of low-risk patients that showed favorable biochemical control, toxicity, and quality of life after five years of follow-up ${ }^{47}$.

\section{II.4 - Treatment techniques}

KQ 11 - Table 6. Is it adequate to perform uHypo with the following techniques?

The panel considered the use of 2D-RT for uHypo inappropriate (level of agreement $-100 \%$, level of evidence - strong).

Table 5. Disease control, toxicity, and quality of life of ultra-hypofractionation.

\begin{tabular}{l|c|c|c}
\hline Outcomes & $\begin{array}{c}\text { Agree, } \\
\text { adequate (\%) }\end{array}$ & $\begin{array}{c}\text { Disagree, } \\
\text { inadequate (\%) }\end{array}$ & Consensus \\
$\begin{array}{l}\text { In terms of disease control, the results of ultra- } \\
\text { hypofractionated radiotherapy are similar to the } \\
\text { conventional fractionation }\end{array}$ & 100 & Yes \\
\hline $\begin{array}{l}\text { In terms of acute urinary toxicity, it is safe to use } \\
\text { ultra-hypofractionation }\end{array}$ & 100 & 0 & Yes \\
\hline $\begin{array}{l}\text { In terms of late urinary toxicity, it is safe to use } \\
\text { ultra-hypofractionation }\end{array}$ & 100 & 0 & Yes \\
\hline $\begin{array}{l}\text { In terms of acute gastrointestinal toxicity, it is safe to use } \\
\text { ultra-hypofractionation }\end{array}$ & 100 & 0 & Yes \\
\hline $\begin{array}{l}\text { In terms of late gastrointestinal toxicity, it is safe to use } \\
\text { ultra-hypofractionation }\end{array}$ & 100 & 0 & Yes \\
\hline $\begin{array}{l}\text { In terms of erectile disfunction, it is safe to use } \\
\text { ultra-hypofractionation }\end{array}$ & 100 & 0 & Yes \\
\hline $\begin{array}{l}\text { In terms of quality of life, ultra-hypofractionation is similar } \\
\text { to conventional fractionation }\end{array}$ & 100 & 0 & \\
\hline
\end{tabular}


Although the majority of panel considered that $3 \mathrm{D}-\mathrm{RT}$ is adequate for uHypo, it did not reach consensus (level of agreement $-68 \%$, level of evidence - medium). Finally, they agreed that intensity modulated techniques (IMRT/VMAT) are adequate for uHypo (level of agreement - 100\%, level of evidence - strong).

Most of the prospective uHypo studies treated patients with IMRT or VMAT. In the Hypo-RT-PC-Trial ${ }^{23}$, however, $80 \%$ of the patients were treated with $3 \mathrm{D}-\mathrm{RT}$, and no difference in toxicity or biochemical control was reported. All patients in the PACE-B trial ${ }^{24}$ were treated with IMRT.

\section{II.5 - Image-guided radiotherapy}

\section{II.5.1 - Modality and frequency of IGRT}

KQ 12 - Table 6. Considering the use of IGRT, which of the following techniques of imaging are adequate for uHypo? Is it mandatory to have daily images?

The panel agreed that the use of tridimensional imaging devices (CB-CT or US) with or without fiducials or bidimensional onboard devices with fiducials are adequate to treat patients with uHypo (level of agreement - 100\%, level of evidence - strong). In addition, other modalities are inappropriate for uHypo, like onboard bidimensional imaging without fiducials or offline bidimensional imaging with or without fiducials (level of agreement - 100\%, level of evidence - medium). Due to the very high dose per fraction, we suggest that treatment must be as fast and accurate as possible to minimize intrafraction errors. Thus, it is mandatory to perform daily image to guide uHypo treatment (level of agreement $-100 \%$, level of evidence - strong).

The Hypo-RT-PC-Trial ${ }^{23}$ allowed IGRT with orthogonal films with fiducials or CB-CT. In the PACE-B trial ${ }^{24}, 55 \%$ of the patients that underwent uHypo were imaged with CB-CT with or without fiducials, $41 \%$ with planar images with intrafraction tracking, and $2.4 \%$ with planar images with fiducials. There are studies comparing the different IGRT techniques, with no significant benefit from one technique over the other ${ }^{52}$.

\subsection{2 - Intrafraction monitoring}

KQ 13 - Table 6. Considering the use of images to guide RT treatment, is it safe to perform uHypo without intrafraction monitoring?

The panel considered safe to carry out uHypo treatment without intra-fraction monitoring (level of agreement - 94\%, level of evidence - medium). The panelists recommended, however, that treatment should be delivered in the shortest possible time to reduce intrafraction errors due to prostate or patient movement, and time length should be remembered and considered by the assisting team.

Table 6. Techniques of ultra-hypofractionation treatment.

\begin{tabular}{l|c|c|c}
\hline Treatment techniques & $\begin{array}{c}\text { Agree, } \\
\text { adequate (\%) }\end{array}$ & $\begin{array}{c}\text { Disagree, } \\
\text { inadequate (\%) }\end{array}$ & $\begin{array}{c}\text { Consensus } \\
\text { achieved }\end{array}$ \\
\hline Planning: conventional or bidimensional radiotherapy & 0 & 100 & Yes \\
\hline Planning: conformal or tridimensional radiotherapy & 68 & 32 & No \\
\hline Planning: intensity modulated techniques (IMRT or VMAT) & 100 & 0 & Yes \\
\hline $\begin{array}{l}\text { Modality of IGRT: tridimensional imaging (cone beam computed } \\
\text { tomography; ultrasonography) with or without fiducials }\end{array}$ & 100 & 0 & Yes \\
\hline $\begin{array}{l}\text { Modality of IGRT: online bidimensional imaging (portal) with } \\
\text { fiducials (onboard imaging, EPID) }\end{array}$ & 100 & 0 & Yes \\
\hline $\begin{array}{l}\text { Modality of IGRT: online bidimensional imaging (portal) } \\
\text { without fiducials (onboard imaging, EPID) }\end{array}$ & 0 & 100 & Yes \\
\hline $\begin{array}{l}\text { Modality of IGRT: offline bidimensional imaging (portal) } \\
\text { with fiducials (analogic portal film or electronic portal film } \\
\text { without onboard device) }\end{array}$ & 0 & 100 & Yes \\
\hline $\begin{array}{l}\text { Modality of IGRT: offline bidimensional imaging (portal) } \\
\text { without fiducials (analogic portal film or electronic portal } \\
\text { film without onboard device) }\end{array}$ & 0 & 100 & Yes \\
\hline \begin{tabular}{l} 
Frequency of IGRT: daily images are mandatory \\
\hline Intrafraction monitoring is mandatory
\end{tabular} & 100 & 0 & Yes \\
\hline
\end{tabular}

IMRT: intensity modulated radiotherapy; VMAT: volumetric modulated arc therapy; IGRT: image-guided radiotherapy; EPID: electronic portal imaging device. 
The Hypo-RT-PC trial $^{23}$ did not use intrafraction monitoring, while the PACE-B trial ${ }^{24}$ used it only in some selected patients. For those without intra-fraction monitoring in the PACE-B trial, static image was repeated in the uHypo arm for treatments extending more than three minutes.

\section{CONCLUSIONS}

The use of hypofractionation has several advantages, such as more convenient schedule and smarter use of RT resources. As a consequence, patient's access to treatment can be increased, which is especially important in LMIC like Brasil. The recommendations given in this paper focused on the acceptable techniques and resources necessary to safely deliver hypofractionated RT for PCa.

In terms of scenarios, the panel agreed that mHypo is adequate for all risk group patients, regardless the treatment of seminal vesicles or pelvic drainage. The most recommended schedule was 60 Gy $(20 \times 3$ Gy). The panel agreed that treatment might be delivered with 3D-RT, but the use of IMRT/VMAT is encouraged. On IGRT, 3D or 2D online images with fiducials can be used with a minimum frequency of three times a week or weekly after five consecutive images. Online portals without fiducials were considered safe for the majority of panelists, but this statement did not reach consensus and is a matter of debate.

For uHypo, the panel agreed that it is effective and safe for low and intermediate risk patients. The treatment might be done regardless the treatment of seminal vesicles. The panel judged inadequate the treatment of pelvic drainage with uHypo. The recommended schedules were 42.7 Gy $(7$ x 6.1 Gy), 40 Gy (5 x 8 Gy) or 36.25 Gy (5 x 7.25 Gy). The panel agreed that uHypo must be delivered using IMRT/VMAT and daily IGRT (3D or 2D online images with fiducials).

This consensus did not address recommendations regarding the definition of target volume, PTV margins, or dose constraints. We advocate that each department must consider its own particularities and protocols of published studies to make the best decisions.

\section{ACKNOWLEDGMENTS}

The SBRT thanks the ABFM and the SBU for their continuous and solid support and partnership. We also thank our secretary assistants, Rafaela Bernardo and Vanessa Carvalho, for their hard work and dedication.

The SBRT thanks Varian Medical Systems Brazil for their financial support used to hold a consensus meeting, which took place on October 11, 2019 in São Paulo.

\section{AUTHORS" CONTRIBUTIONS}

DMFP: Conceptualization, Data Curation, Formal Analysis, Investigation, Methodology, Project Administration, Validation, Visualization, Writing - Original Draft, Writing - Review \& Editing. LCFP: Conceptualization, Data Curation, Formal Analysis, Investigation, Methodology, Project Administration, Resources, Supervision, Validation, Visualization, Writing - Original Draft, Writing - Review \& Editing. MSC: Conceptualization, Data Curation, Formal Analysis, Investigation, Methodology, Project Administration, Resources, Supervision, Validation, Visualization, Writing - Original Draft, Writing - Review \& Editing. ABC: Conceptualization, Data Curation, Formal Analysis, Investigation, Methodology, Project Administration, Validation, Visualization, Writing - Original Draft, Writing - Review \& Editing. MLR: Conceptualization, Data Curation, Formal Analysis, Investigation, Methodology, Project Administration, Validation, Visualization, Writing - Original Draft, Writing - Review \& Editing. FQK: Writing-Original Draft, Writing-Review \& Editing. AP: Writing - Review \& Editing. ETTL: Writing - Review \& Editing. FLCF: Writing - Review \& Editing. FMO: Writing - Review \& Editing. FNBBFC: Writing - Review \& Editing. GTP: Writing - Review \& Editing. ITC: Writing - Review \& Editing. JLFS: Writing Review \& Editing. LKKM: Writing - Review \& Editing. PHRZ: Writing - Review \& Editing. RMH: Writing - Review \& Editing. AAR: Conceptualization, Data Curation, Formal Analysis, Funding Acquisition, Investigation, Methodology, Project Administration, Resources, Supervision, Validation, Visualization.

\section{REFERENCES}

1. INCA - Instituto Nacional de Câncer - Estimativa 2018 [Internet]. [cited on Nov 30, 2019]. Available from: http://www1.inca. gov.br/estimativa/2018/.

2. Bray F, Ferlay J, Soerjomataram I, Siegel RL, Torre LA, Jemal A. Global cancer statistics 2018: GLOBOCAN estimates of incidence and mortality worldwide for 36 cancers in 185 countries. CA Cancer J Clin. 2018;68(6):394-424. https://doi.org/10.3322/caac.21492

3. Gay HA, Michalski JM. Radiation therapy for prostate cancer. Mo Med. 2018;115:146-50. PMID: 30228707
4. Burt LM, Shrieve DC, Tward JD. Factors influencing prostate cancer patterns of care: an analysis of treatment variation using the SEER database. Adv Radiat Oncol 2018;3(2):17080. https://doi.org/10.1016/j.adro.2017.12.008

5. Ministério da Saúde, Secretaria de Atenção Especializada em Saúde, Departamento de Atenção Especializada e Temática. Censo radioterapia [Internet]. [cited on April 20, 2020]. Available from: https://portalarquivos2.saude.gov.br/images/ pdf/2019/julho/26/paper-radioterapia-ALT3.pdf. 
6. Datta NR, Stutz E, Rogers S, Bodis S. Conventional versus hypofractionated radiation therapy for localized or locally advanced prostate cancer: a systematic review and metaanalysis along with therapeutic implications. Int J Radiat Oncol Biol Phys. 2017;99(3):573-89. https://doi.org/10.1016/j. ijrobp.2017.07.021

7. Arcangeli S, Greco C. Hypofractionated radiotherapy for organ-confined prostate cancer: is less more? Nat Rev Urol. 2016;13(7):400-8. https://doi.org/10.1038/nrurol.2016.106

8. Arcangeli G, Arcangeli S, Pinzi V, Benassi M, Benassi M, Strigari L. Optimal scheduling of hypofractionated radiotherapy for localized prostate cancer: A systematic review and metanalysis of randomized clinical trials. Cancer Treat Rev. 2018;70:22-9. https://doi.org/10.1016/j.ctrv.2018.07.003

9. Dearnaley D, Syndikus I, Mossop H, Khoo V, Birtle A, Bloomfield $D$, et al. Conventional versus hypofractionated high-dose intensity-modulated radiotherapy for prostate cancer: 5-year outcomes of the randomised, non-inferiority, phase $3 \mathrm{CHHiP}$ trial. Lancet Oncol. 2016;17:1047-60. https://doi.org/10.1016/ S1470-2045(16)30102-4

10. Wilkins A, Mossop H, Syndikus I, Khoo V, Bloomfield D, Parker $C$, et al. Hypofractionated radiotherapy versus conventionally fractionated radiotherapy for patients with intermediate-risk localised prostate cancer: 2-year patient-reported outcomes of the randomised, non-inferiority, phase $3 \mathrm{CHHiP}$ trial. Lancet Oncol. 2015;16:1605-16. https://doi.org/10.1016/S1470-2045(15)00280-6

11. Aluwini S, Pos F, Schimmel E, van Lin E, Krol S, van der Toorn $\mathrm{PP}$, et al. Hypofractionated versus conventionally fractionated radiotherapy for patients with prostate cancer (HYPRO): acute toxicity results from a randomised non-inferiority phase 3 trial. Lancet Oncol. 2015;16(3):274-83. https://doi.org/10.1016/ S1470-2045(14)70482-6

12. Aluwini S, Pos F, Schimmel E, Krol S, van der Toorn PP, de Jager $\mathrm{H}$, et al. Hypofractionated versus conventionally fractionated radiotherapy for patients with prostate cancer (HYPRO): late toxicity results from a randomised, non-inferiority, phase 3 trial. Lancet Oncol. 2016:17(4):464-74. https://doi.org/10.1016/ S1470-2045(15)00567-7

13. Incrocci L, Wortel RC, Alemayehu WG, Aluwini S, Schimmel E, Krol S, et al. Hypofractionated versus conventionally fractionated radiotherapy for patients with localised prostate cancer (HYPRO): final efficacy results from a randomised, multicentre, open-label, phase 3 trial. Lancet Oncol. 2016;17(8):1061-9. https://doi.org/10.1016/\$1470-2045(16)30070-5

14. Catton CN, Lukka H, Gu CS, Martin JM, Supiot S, Chung PWM, et al. Randomized Trial of a Hypofractionated Radiation Regimen for the Treatment of Localized Prostate Cancer. J Clin Oncol. 2017;35(17):1884-90. https://doi.org/10.1200/JCO.2016.71.7397

15. Lee WR, Dignam JJ, Amin MB, Bruner DW, Low D, Swanson $\mathrm{GP}$, et al. Randomized phase III noninferiority study comparing two radiotherapy fractionation schedules in patients with low-risk prostate cancer. J Clin Oncol. 2016;34(20):2325-32. https://doi.org/10.1200/JCO.2016.67.0448

16. Bruner DW, Pugh SL, Lee WR, Hall WA, Dignam JJ, Low D, et al. Quality of life in patients with low-risk prostate cancer treated with hypofractionated vs conventional radiotherapy: a phase 3 randomized clinical trial. JAMA Oncol. 2019;5(5):66470. https://doi.org/10.1001/jamaoncol.2018.6752

17. Pollack A, Walker G, Horwitz EM, Price R, Feigenberg S, Konski AA, et al. Randomized trial of hypofractionated external-beam radiotherapy for prostate cancer. J Clin Oncol. 2013;31(31):3860-8. https://doi.org/10.1200/JCO.2013.51.1972
18. Shaikh T, Li T, Handorf EA, Johnson ME, Wang LS, Hallman $M A$, et al. Long-Term patient-reported outcomes from a phase 3 randomized prospective trial of conventional versus hypofractionated radiation therapy for localized prostate cancer. Int J Radiat Oncol Biol Phys. 2017;97:722-31. https:// doi.org/ 10.1016/j.jirobp.2016.12.034

19. Hoffman KE, Skinner $H$, Pugh TJ, Voong KR, Levy LB, Choi $S$, et al. Patient-reported Urinary, bowel, and sexual function after hypofractionated intensity-modulated radiation therapy for prostate cancer: results from a randomized trial. Am J Clin Oncol. 2018;41(6):558-67. https://doi.org/10.1097/ COC. 0000000000000325

20. Hoffman KE, Voong KR, Pugh TJ, Skinner H, Levy LB, Takiar $V$, et al. Risk of late toxicity in men receiving dose-escalated hypofractionated intensity modulated prostate radiation therapy: results from a randomized trial. Int J Radiat Oncol Biol Phys. 2014;88(5):1074-84. https://doi.org/10.1016/j.jrobp.2014.01.015

21. Hoffman KE, Voong KR, Levy LB, Allen PK, Choi S, Schlembach $\mathrm{PJ}$, et al. Randomized trial of hypofractionated, dose-escalated, intensity-modulated radiation therapy (imrt) versus conventionally fractionated imrt for localized prostate cancer. J Clin Oncol. 2018;36(29):2943-9. https://doi.org/10.1200/JCO.2018.77.9868.

22. Arcangeli G, Saracino B, Arcangeli S, Gomellini S, Petrongari $M G$, Sanguineti $G$, et al. Moderate hypofractionation in highrisk, organ-confined prostate cancer: final results of a phase iii randomized trial. J Clin Oncol. 2017;35(17):1891-7. https:// doi.org/10.1200/JCO.2016.70.4189

23. Widmark A, Gunnlaugsson A, Beckman L, Thellenberg-Karlsson C, Hoyer M, Lagerlund M, et al. Ultra-hypofractionated versus conventionally fractionated radiotherapy for prostate cancer: 5 -year outcomes of the HYPO-RT-PC randomised, non-inferiority, phase 3 trial. The Lancet. 2019;394(10196):385-95. https:// doi.org/10.1016/S0140-6736(19)31131-6

24. Brand DH, Tree AC, Ostler P, van der Voet $\mathrm{H}$, Loblaw A, Chu $W$, et al. Intensity-modulated fractionated radiotherapy versus stereotactic body radiotherapy for prostate cancer (PACE-B): acute toxicity findings from an international, randomised, open-label, phase 3, non-inferiority trial. Lancet Oncol. 2019;20(11):153143. https://doi.org/10.1016/S1470-2045(19)30569-8

25. Mangoni M, Desideri I, Detti B, Bonomo P, Greto D, Paiar F, et al. Hypofractionation in prostate cancer: radiobiological basis and clinical appliance. BioMed Res Int. 2014;2014:781340. https://doi.org/10.1155/2014/781340

26. Glazebrook GA. Radiation therapy: a long term cost benefit analysis in a North American region. Clin Oncol (R Coll Radiol). 1992;4(5):302-5. https://doi.org/10.1016/s0936-6555(05)81105-7

27. Moraes FY de, Marta GN, Hanna SA, Leite ET, Ferrigno R, da Silva JL, et al. Brazil's challenges and opportunities. Int J Radiat Oncol Biol Phys. 2015;92(4):707-12. https://doi.org/10.1016/j. ijrobp.2014.12.063

28. Weltman E, Marta GN. Radiotherapy and the SUS: A collapse foretold. Rev Assoc Med Bras (1992). 2017;63(2):93-4. https:// doi.org/10.1590/1806-9282.63.02.93

29. Moraes FY, Mendez LC, Rosa AA, Marta GN. Expanding access to radiation therapy: an update on Brazil's current challenges and opportunities. Int J Radiat Oncol Biol Phys. 2018;102(2):463-4. https://doi.org/10.1016/j.ijrobp.2018.05.003

30. Sinha IP, Smyth RL, Williamson PR. Using the Delphi Technique to Determine Which Outcomes to Measure in Clinical Trials: Recommendations for the Future Based on a Systematic Review of Existing Studies. PLoS Med. 2011;8(1):e1000393. https://doi.org/10.1371/journal.pmed.1000393 
31. Devisetty K, Jani AB, Choe KS, Liauw SL. Should prior TURP influence the decision to treat prostate cancer with external beam radiation therapy? Int J Radiat Oncol Biol Phys. 2007;69(3):S360-1. https://doi.org/10.1016/j.jijrobp.2007.07.1456

32. Devisetty K, Zorn KC, Katz MH, Jani AB, Liauw SL. External beam radiation therapy after transurethral resection of the prostate: a report on acute and late genitourinary toxicity. 2010;77(4):1060-5. https://doi.org/10.1016/j.jirobp.2009.06.078

33. Norkus D, Karklelyte A, Engels B, Versmessen H, Griskevicius R, De Ridder M. A randomized hypofractionation dose escalation trial for high risk prostate cancer patients: interim analysis of acute toxicity and quality of life in 124 patients. Radiat Oncol. 2013;8:206. https://doi.org/10.1186/1748-717X-8-206

34. Viani GA, Viana BS, Martin JEC, Rossi BT, Zuliani G, Stefano EJ. Intensity-modulated radiotherapy reduces toxicity with similar biochemical control compared with 3-dimensional conformal radiotherapy for prostate cancer: a randomized clinical trial. Cancer. 2016;122(13):2004-11. https://doi.org/10.1002/cncr.29983

35. Al-Mamgani A, Heemsbergen WD, Peeters STH, Lebesque JV. Role of intensity-modulated radiotherapy in reducing toxicity in dose escalation for localized prostate cancer. Int J Radiat Oncol Biol Phys. 2009;73(3):685-91. https://doi.org/10.1016/j.jirobp.2008.04.063

36. Michalski JM, Yan Y, Watkins-Bruner D, Bosch WR, Winter K, Galvin JM, et al. Preliminary toxicity analysis of 3-dimensional conformal radiation therapy versus intensity modulated radiation therapy on the high-dose arm of the Radiation Therapy Oncology Group 0126 prostate cancer trial. Int J Radiat Oncol Biol Phys. 2013;87(5)932-8. https://doi.org/10.1016/j.jirobp.2013.07.041

37. Zelefsky MJ, Levin EJ, Hunt M, Yamada Y, Shippy AM, Jackson $A$, et al. Incidence of late rectal and urinary toxicities after threedimensional conformal radiotherapy and intensity-modulated radiotherapy for localized prostate cancer. Int J Radiat Oncol Biol Phys. 2008;70(4):1124-9. https://doi.org/10.1016/j.jirobp.2007.11.044

38. de Crevoisier R, Bayar MA, Pommier P, Muracciole X, Pêne F, Dudouet $P$, et al. Daily versus weekly prostate cancer image guided radiation therapy: phase 3 multicenter randomized trial. Int J Radiat Oncol Biol Phys. 2018;102(5):1420-9. https:// doi.org/10.1016/j.ijrobp.2018.07.2006

39. Zelefsky MJ, Kollmeier M, Cox B, Fidaleo A, Sperling D, Pei X, et al. Improved clinical outcomes with high-dose image guided radiotherapy compared with non-IGRT for the treatment of clinically localized prostate cancer. Int J Radiat Oncol Biol Phys. 2012;84(1):125-9. https://doi.org/10.1016/j.jjrobp.2011.11.047

40. Valeriani M, Bracci S, Osti MF, Falco T, Agolli L, De Sanctis V, et al. Intermediate-risk prostate cancer patients treated with androgen deprivation therapy and a hypofractionated radiation regimen with or without image guided radiotherapy. Radiat Oncol. 2013;8:137. https://doi.org/10.1186/1748-717X-8-137

41. Chung HT, Xia P, Chan LW, Park-Somers E, Roach M 3rd. Does image-guided radiotherapy improve toxicity profile in whole pelvic-treated high-risk prostate cancer? Comparison between IG-IMRT and IMRT. Int J Radiat Oncol Biol Phys. 2009;73(1):5360. https://doi.org/10.1016/j.jjrobp.2008.03.015

42. Wortel RC, Incrocci L, Pos FJ, Lebesque JV, Witte MG, van der Heide UA, et al. Acute toxicity after image-guided intensity modulated radiation therapy compared to 3D conformal radiation therapy in prostate cancer patients. Int J Radiat Oncol Biol Phys. 2015;91(4):737-44. https://doi.org/10.1016/j.jjrobp.2014.12.017
43. Sveistrup J, af Rosenschöld PM, Deasy JO, Oh JH, Pommer T, Petersen PM, et al. Improvement in toxicity in high risk prostate cancer patients treated with image-guided intensity-modulated radiotherapy compared to 3D conformal radiotherapy without daily image guidance. Radiat Oncol. 2014;9:44. https://doi. org/10.1186/1748-717X-9-44

44. Zapatero A, Roch $M$, Büchser D, Castro P, Fernández-Banda L, Pozo G, et al. Reduced late urinary toxicity with high-dose intensity-modulated radiotherapy using intra-prostate fiducial markers for localized prostate cancer. Clin Transl Oncol. 2017;19(9):1161-7. https://doi.org/10.1007/s12094-017$1655-9$

45. Becker-Schiebe M, Abaci A, Ahmad T, Hoffmann W. Reducing radiation-associated toxicity using online image guidance (IGRT) in prostate cancer patients undergoing dose-escalated radiation therapy. Rep Pract Oncol Radiother. 2016;21(3):188-94. https://doi.org/10.1016/j. rpor.2016.01.005

46. Kok D, Gill S, Bressel M, Byrne K, Kron T, Fox C, et al. Late toxicity and biochemical control in 554 prostate cancer patients treated with and without dose escalated image guided radiotherapy. Radiother Oncol. 2013;107(2):140-6. https://doi.org/10.1016/j.radonc.2013.04.007

47. Mantz C. A Phase II Trial of Stereotactic Ablative Body Radiotherapy for Low-Risk Prostate Cancer Using a Non-Robotic Linear Accelerator and Real-Time Target Tracking: Report of Toxicity, Quality of Life, and Disease Control Outcomes with 5-Year Minimum Follow-Up. Front Oncol. 2014;4:279. https:// doi.org/10.3389/fonc.2014.00279

48. Hannan R, Tumati V, Xie X-J, Cho LC, Kavanagh BD, Brindle $J$, et al. Stereotactic body radiation therapy for low and intermediate risk prostate cancer-Results from a multiinstitutional clinical trial. Eur J Cancer. 2016;59:142-51. https:// doi.org/10.1016/j.ejca.2016.02.014

49. Musunuru HB, Quon $H$, Davidson $M$, Cheung $P$, Zhang $L$, D'Alimonte $L$, et al. Dose-escalation of five-fraction SABR in prostate cancer: Toxicity comparison of two prospective trials. Radiother Oncol. 2016;118(1):112-7. https://doi.org/10.1016/j. radonc.2015.12.020

50. Zimmermann M, Taussky D, Menkarios C, Vigneault É, Beauchemin MC, Bahary JP, et al. Prospective phase II trial of once-weekly hypofractionated radiation therapy for low-risk adenocarcinoma of the prostate: late toxicities and outcomes. Clin Oncol (R Coll Radiol). 2016;28(6):386-92. https://doi. org/10.1016/j.clon.2015.12.024

51. Jackson WC, Silva J, Hartman HE, Dess RT, Kishan AU, Beeler $\mathrm{WH}$, et al. Stereotactic body radiation therapy for localized prostate cancer: a systematic review and meta-analysis of over 6,000 patients treated on prospective studies. Int J Radiat Oncol Biol Phys. 2019;104(4):778-9. https://doi.org/10.1016/j. ijrobp.2019.03.051

52. Moseley DJ, White EA, Wiltshire KL, Rosewall T, Sharpe $M B$, Siewerdsen JH, et al. Comparison of localization performance with implanted fiducial markers and conebeam computed tomography for on-line image-guided radiotherapy of the prostate. Int J Radiat Oncol Biol Phys. 2007;67(3):942-53. https://doi.org/10.1016/j. ijrobp.2006.10.039 


\section{ERRATUM}

https://doi.org/10.1590/1806-9282.67.01.002ERRATUM

In the manuscript "Hypofractionated radiotherapy recommendations for localized prostate cancer in Brasil", DOI: 10.1590/18069282.67.01.002, published in the Rev Assoc Med Bras. 2021;67(1):7-18.

Page 7, title:

Where it reads:

Hypofractionated radiotherapy recommendations for localized prostate cancer in Brasil

It should read:

Hypofractionated radiotherapy recommendations for localized prostate cancer in Brazil

Page 7, summary

Where it reads:

There are many benefits of hypofractionation, including a more convenient schedule for the patients and better use of resources, which is especially important in low- and middle-income countries like Brasil. Based on these data, the Brazilian Society of Radiotherapy (Sociedade Brasileira de Radioterapia) organized this consensus to guide and support the use of hypofractionated radiotherapy for localized prostate cancer in Brasil. METHODS: The relevant literature regarding moderate hypofractionation (mHypo) and ultra-hypofractionation (uHypo) was reviewed and discussed by a group of experts from public and private centers of different parts of Brasil.

\section{It should read:}

There are many benefits of hypofractionation, including a more convenient schedule for the patients and better use of resources, which is especially important in low- and middle-income countries like Brazil. Based on these data, the Brazilian Society of Radiotherapy (Sociedade Brasileira de Radioterapia) organized this consensus to guide and support the use of hypofractionated radiotherapy for localized prostate cancer in Brazil. METHODS: The relevant literature regarding moderate hypofractionation (mHypo) and ultra-hypofractionation (uHypo) was reviewed and discussed by a group of experts from public and private centers of different parts of Brazil.

Page 7, institutional bond

\section{Where it reads:}

${ }^{1}$ Hospital Sírio-Libanês - Brasília (DF), Brasil.

${ }^{2}$ Hospital Felício Rocho - Belo Horizonte (MG), Brasil.

${ }^{3}$ Hospital Mãe de Deus - Porto Alegre (RS), Brasil.

${ }^{4}$ Americas Centro de Oncologia Integrado - Rio de Janeiro (RJ), Brasil.

${ }^{5}$ Hospital São José - Criciúma (SC), Brasil.

${ }^{6}$ Hospital da Providência - Apucarana (PR), Brasil

${ }^{7}$ Universidade de Sáo Paulo, Hospital Vila Nova Star, Rede D’Or, Instituto do Câncer do Estado de São Paulo - São Paulo (SP), Brasil. ${ }^{8}$ Hospital de Amor de Barretos - Barretos (SP), Brasil.

${ }^{9}$ Grupo Oncoclínicas, Hospital São Lucas Pontifícia Universidade Católica do Rio Grande do Sul - Porto Alegre (RS), Brasil.

${ }^{10}$ Hospital São Domingos - São Luís (MA), Brasil.

${ }^{11}$ Santa Casa de Misericórdia, Hospital Moinhos de Vento - Porto Alegre (RS), Brasil.

${ }^{12}$ Hospital Israelita Albert Einstein - São Paulo (SP), Brasil.

${ }^{13}$ Hospital Sírio-Libanês - Sáo Paulo (SP), Brasil.

${ }^{14}$ Grupo Oncologia D’Or, Clínica São Vicente - Rio de Janeiro (RJ), Brasil.

${ }^{15}$ Hospital Beneficência Portuguesa - São Paulo (SP), Brasil.

${ }^{16}$ Hospital Alemão Oswaldo Cruz - São Paulo (SP), Brasil.

${ }^{17}$ Sociedade Brasileira de Radioterapia, Grupo Oncoclínicas - São Paulo (SP), Brasil. 


\section{It should read:}

${ }^{1}$ Hospital Sírio-Libanês - Brasília (DF), Brazil.

${ }^{2}$ Hospital Felício Rocho - Belo Horizonte (MG), Brazil.

${ }^{3}$ Hospital Mãe de Deus - Porto Alegre (RS), Brazil.

${ }^{4}$ Americas Centro de Oncologia Integrado - Rio de Janeiro (RJ), Brazil.

${ }^{5}$ Hospital São José - Criciúma (SC), Brazil.

${ }^{6}$ Hospital da Providência - Apucarana (PR), Brazil

${ }^{7}$ Universidade de São Paulo, Hospital Vila Nova Star, Rede D’Or, Instituto do Câncer do Estado de São Paulo - São Paulo (SP), Brazil. ${ }^{8}$ Hospital de Amor de Barretos - Barretos (SP), Brazil.

${ }^{9}$ Grupo Oncoclínicas, Hospital Sáo Lucas Pontifícia Universidade Católica do Rio Grande do Sul - Porto Alegre (RS), Brazil.

${ }^{10}$ Hospital São Domingos - São Luís (MA), Brazil.

${ }^{11}$ Santa Casa de Misericórdia, Hospital Moinhos de Vento - Porto Alegre (RS), Brazil.

${ }^{12}$ Hospital Israelita Albert Einstein - São Paulo (SP), Brazil.

${ }^{13}$ Hospital Sírio-Libanês - São Paulo (SP), Brazil.

${ }^{14}$ Grupo Oncologia D’Or, Clínica São Vicente - Rio de Janeiro (RJ), Brazil.

${ }^{15}$ Hospital Beneficência Portuguesa - São Paulo (SP), Brazil.

${ }^{16}$ Hospital Alemão Oswaldo Cruz - São Paulo (SP), Brazil.

${ }^{17}$ Sociedade Brasileira de Radioterapia, Grupo Oncoclínicas - São Paulo (SP), Brazil.

Page 8, Introduction, first column, first paragraph

Where it reads:

Prostate cancer $(\mathrm{PCa})$ is one of the most incident cancers in Brasil and worldwide, representing more than $30 \%$ of all cases in men ${ }^{1,2}$.

\section{It should read:}

Prostate cancer (PCa) is one of the most incident cancers in Brazil and worldwide, representing more than $30 \%$ of all cases in men ${ }^{1,2}$.

Page 8, Introduction, second column, first paragraph

\section{Where it reads:}

In order to support radiation oncologists and physicists to implement hypofractionation in the clinical practice, this consensus aimed to guide indications and the minimum requirements to safely conduct hypofractionation RT for localized PCa patients in Brasil.

\section{It should read:}

In order to support radiation oncologists and physicists to implement hypofractionation in the clinical practice, this consensus aimed to guide indications and the minimum requirements to safely conduct hypofractionation RT for localized PCa patients in Brazil.

Page 8, Methods, second column, second paragraph

\section{Where it reads:}

Sixteen radiation oncologists from different areas of Brasil, from both public and private institutions, with known expertise in the topic, attended the meeting and composed the panel.

\section{It should read:}

Sixteen radiation oncologists from different areas of Brazil, from both public and private institutions, with known expertise in the topic, attended the meeting and composed the panel. 
Page 11, Table 3

Where it reads:

Table 3. Techniques of moderate hypofractionation treatment.

\begin{tabular}{|c|c|c|c|}
\hline Treatment techniques & $\begin{array}{l}\text { Yes, adequate } \\
(\%)\end{array}$ & $\begin{array}{l}\text { No, inadequate } \\
(\%)\end{array}$ & $\begin{array}{l}\text { Consensus } \\
\text { achieved }\end{array}$ \\
\hline Planning: conventional or bidimensional radiotherapy & 0 & 100 & Yes \\
\hline Planning: conformal or tridimensional radiotherapy & 88 & 12 & Yes \\
\hline Planning: intensity modulated techniques (IMRT or VMAT) & 100 & 0 & Yes \\
\hline $\begin{array}{l}\text { Modality of IGRT: tridimensional imaging (cone beam computed } \\
\text { tomography; ultrasonography) with or without fiducials }\end{array}$ & 100 & 0 & Yes \\
\hline $\begin{array}{l}\text { Modality of IGRT: online bidimensional imaging (portal) with } \\
\text { fiducials (onboard imaging, EPID) }\end{array}$ & 100 & 0 & Yes \\
\hline $\begin{array}{l}\text { Modality of IGRT: online bidimensional imaging (portal) without } \\
\text { fiducials (onboard imaging, EPID) }\end{array}$ & 69 & 31 & Yes \\
\hline
\end{tabular}

\section{It should read:}

Table 3. Techniques of moderate hypofractionation treatment.

\begin{tabular}{|c|c|c|c|}
\hline Treatment techniques & $\begin{array}{l}\text { Yes, adequate } \\
(\%)\end{array}$ & $\begin{array}{l}\text { No, inadequate } \\
(\%)\end{array}$ & $\begin{array}{l}\text { Consensus } \\
\text { achieved }\end{array}$ \\
\hline Planning: conventional or bidimensional radiotherapy & 0 & 100 & Yes \\
\hline Planning: conformal or tridimensional radiotherapy & 88 & 12 & Yes \\
\hline Planning: intensity modulated techniques (IMRT or VMAT) & 100 & 0 & Yes \\
\hline $\begin{array}{l}\text { Modality of IGRT: tridimensional imaging (cone beam computed } \\
\text { tomography; ultrasonography) with or without fiducials }\end{array}$ & 100 & 0 & Yes \\
\hline $\begin{array}{l}\text { Modality of IGRT: online bidimensional imaging (portal) with } \\
\text { fiducials (onboard imaging, EPID) }\end{array}$ & 100 & 0 & Yes \\
\hline $\begin{array}{l}\text { Modality of IGRT: online bidimensional imaging (portal) without } \\
\text { fiducials (onboard imaging, EPID) }\end{array}$ & 69 & 31 & No \\
\hline
\end{tabular}

Page 16, Conclusions, first column, second paragraph

Where it reads:

As a consequence, patient's access to treatment can be increased, which is especially important in LMIC like Brasil.

\section{It should read:}

As a consequence, patient's access to treatment can be increased, which is especially important in LMIC like Brazil.

Page 16, Acknowledgments, second column, second paragraph

Where it reads:

The SBRT thanks Varian Medical Systems Brasil for their financial support used to hold a consensus meeting, which took place on October 11, 2019 in São Paulo.

\section{It should read:}

The SBRT thanks Varian Medical Systems Brazil for their financial support used to hold a consensus meeting, which took place on October 11, 2019 in São Paulo. 